\title{
On the horizontal branch of the galactic globular cluster NGC 2808
}

\author{
V. Castellani ${ }^{1,2}$, G. Iannicola ${ }^{1}$, G. Bono ${ }^{1}$, M. Zoccali ${ }^{3}$, S. Cassisi ${ }^{4}$, and R. Buonanno ${ }^{5}$ \\ 1 INAF-Osservatorio Astronomico di Roma, via Frascati 33, 00040 Monte Porzio Catone, Italy \\ e-mail: vittorio@mporzio.astro.it \\ 2 INFN-Sezione di Ferrara, via Paradiso 12, 44100 Ferrara, Italy \\ 3 Universidad Catolica de Chile, Department of Astronomy \& Astrophysics, Casilla 306, Santiago 22, Chile \\ 4 INAF-Osservatorio Astronomico di Teramo, via M. Maggini, 64100 Teramo, Italy \\ 5 Dipartimento di Fisica, Università di Roma Tor Vergata, via della Ricerca Scientifica 1, 00133 Rome, Italy
}

Received 13 October 2004 / Accepted 12 May 2005

\section{ABSTRACT}

\begin{abstract}
We present new UV $(F 218 W)$ data for stars in the central region of the Galactic globular cluster NGC 2808, collected with the WFPC2 camera on board the Hubble Space Telescope. These data together with $F 439 \mathrm{~W}$ and $F 555 \mathrm{~W}$-band data and previous ground-based observations provide a multifrequency coverage of the cluster stellar population extending up to a distance of 1.7 times the cluster core radius. We discuss this complete sample of stars, which includes 764 Red Giant Branch (RGB) stars brighter than the Horizontal Branch (HB) luminosity level, 1239 HB stars, 119 Asymptotic Giant Branch (AGB), and 22 AGB-manqué stellar structures. As already known, we find that blue HB stars separate into three distinct groups. However, our multiband photometry indicates that several stars in the two hotter HB groups show a flat spectrum, thus suggesting the binarity of these objects. Artificial star experiments suggest that at most $50 \%$ of them might be photometric blends. Moreover, at variance with previous claims one finds that canonical Zero Age Horizontal Branch (ZAHB) models do reach effective temperatures typical of observed hot HB stars. We also show that the ratio between HB and RGB stars brighter than the HB luminosity level steadly increases when moving from the cluster center to the periphery, passing from $R=1.37 \pm 0.14$ in the cluster core to $R=1.95 \pm 0.26$ in the outer cluster regions. We discuss the possible origin of such a radial gradient in the context of the Blue Tails phenomenon, advancing some suggestions concerning the clumpy stellar distribution along the HB.
\end{abstract}

Key words. globular clusters: individual: NGC 2808 - stars: evolution - stars: horizontal-branch - global clusters: general

\section{Introduction}

For about thirty years NGC 2808 has been a special conundrum for stellar evolution understanding in Galactic Globular Clusters (GGCs). In 1974 Harris revealed the unexpected bimodal distribution of HB stars in NGC 2808. He found that HB stars populate two well-separated regions of the branch, either to the red or to the blue of the RR Lyrae instability strip. At that time, it was already clear that such an odd occurrence could be easily understood in terms of a bimodal distribution of HB masses and, thus, of mass loss in the HB progenitors, the origin of such bimodality remaining, however, unknown. Owing to its peculiar HB morphology, among the known GGCs, it was dubbed anomalous by Harris (1978).

This empirical scenario became even more puzzling a few years ago, when Sosin et al. (1997) took advantage of data collected with the Wide Field Planetary Camera 2 (WFPC2) on board the Hubble Space Telescope (HST) to provide a deep color magnitude diagram (CMD) of the cluster. They found in the hot portion of the $\mathrm{HB}$ a long blue tail extending over more than $4 V$-magnitudes, with two additional narrow but well-defined gaps. More recently, Sohn et al. (1998) detected in this cluster a significant color gradient, the mean color becoming redder when moving toward the cluster center. This is possibly connected to the origin of the hot HB tail. However, further photometric investigations (Walker 1999; Bedin et al. 2000) failed to find any firm evidence of radial gradients across the cluster in the different evolutionary phases.

To investigate this issue in more detail, and, in general, to reach a better knowledge of the cluster $\mathrm{HB}$ population, in this paper we make use of the $F 218 W$ filter of the WFPC 2 on board HST to secure a robust detection of the faintest blue HB objects in the crowded cluster central region. On this basis, we will discuss the evolutionary status of hot HB stars, presenting evidence for the occurrence in the cluster of a population gradient. Even though population gradients represented the original goal of our investigation, we found that multiband photometric data are of great relevance to investigate the nature of hot HB structures. The present data suggest that a substantial fraction of subluminous HB stars might be binary systems. Taking into account this new empirical evidence, in the final section of this paper we will revisit the problem of multimodal HB distributions, suggesting some possible evolutionary scenarios. 

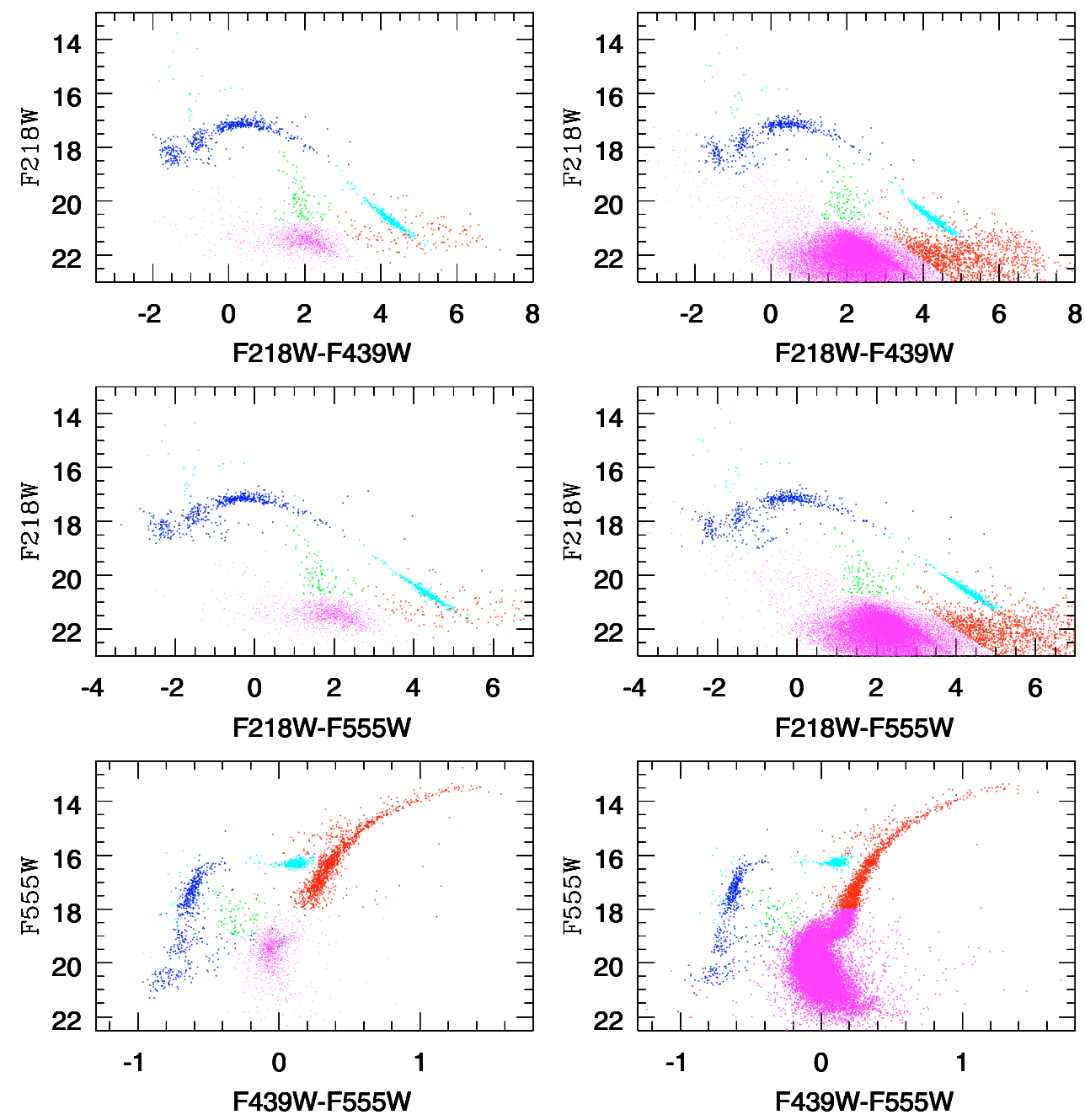

Fig. 1. Optical-UV Color-Magnitude Diagrams derived using two different reduction packages, ROMAFOT (left), and DAOPHOT (right) as well as two different reduction strategies concerning the identification of individual sources. See text for details. (This figure is available in colour in electronic form.)

\section{Observations and data reduction}

We refer to the observational material collected by Bedin et al. (2000), based on data taken with $1.54 \mathrm{~m}$ ESO-Danish Telescope in the optical bands $U, B, V, I$, complemented with similar data from the ESO-NTT Telescope and with $F 439 W$ and $F 555 W$ frames taken with the WFPC2 on board HST (Piotto et al. 2002). In the cluster external region ( $r \geq$ 120 arcsec) unaffected by the crowding of stellar images, we will rely on the Bedin et al. (2000) photometry. Data for the inner region have been reprocessed, with the additional use of $\mathrm{UV}$ data collected with the $F 218 \mathrm{~W}$ filter. The optical and UV data retrieved from the HST archive (ID: GO6095, PI: Sosin) are the following: $F 218 W(1600,1700 \mathrm{~s}), F 439 W(50,2 \times 230 \mathrm{~s})$, and $F 555 W(7,50 \mathrm{~s})$.

For data reduction we adopted the ROMAFOT package (Buonanno \& Iannicola 1989). Even though this package is more demanding than DAOPHOT in terms of labour, it allows an interactive check of the detection and fit accuracy of individual stars in crowded fields. The search was performed on the $F 218 \mathrm{~W}$ data, by adopting a detection threshold of $5 \sigma$ above the sky level. As discussed, e.g. by Ferraro et al. (1999), the advantage of using the UV band is that hot HB stars appear as bright objects, and the images, even in the very center of the cluster, are marginally affected by the crowding of luminous Red Giants. However, this approach also means that the detection of Red Giant stars in UV data is very challenging. To collect a complete sample of cluster luminous stars, the above search was complemented with an additional search on the $F 439 \mathrm{~W}$ data, but only for sources brighter than $F 555 W \sim 17$. The photometry was eventually performed using an analytical PSF, a Moffat function with $\beta=2$, modeled from a sample of $\sim 30$ isolated stars uniformly distributed across the field.

As a test, we repeated the reduction with DAOPHOT/ALLFRAME (Stetson 1987, 1994) as explained in Piotto et al. (2002). The search, in this case, was performed on the median of the $F 439 \mathrm{~W}$ and $F 555 \mathrm{~W}$ images, and then PSF fitting photometry was performed simultaneously on all the frames. The instrumental magnitudes were transformed into the so-called STMAG system of the WFPC2 using the calibration provided by Holtzman et al. (1995).

Figure 1 shows the CMDs obtained using ROMAFOT (left panels) or DAOPHOT/ALLFRAME (right panels). As a consequence of the different reduction strategies, the number of faint cool sources $(F 218 W \geq 22, F 218 W-F 439 W \approx 2)$ is 

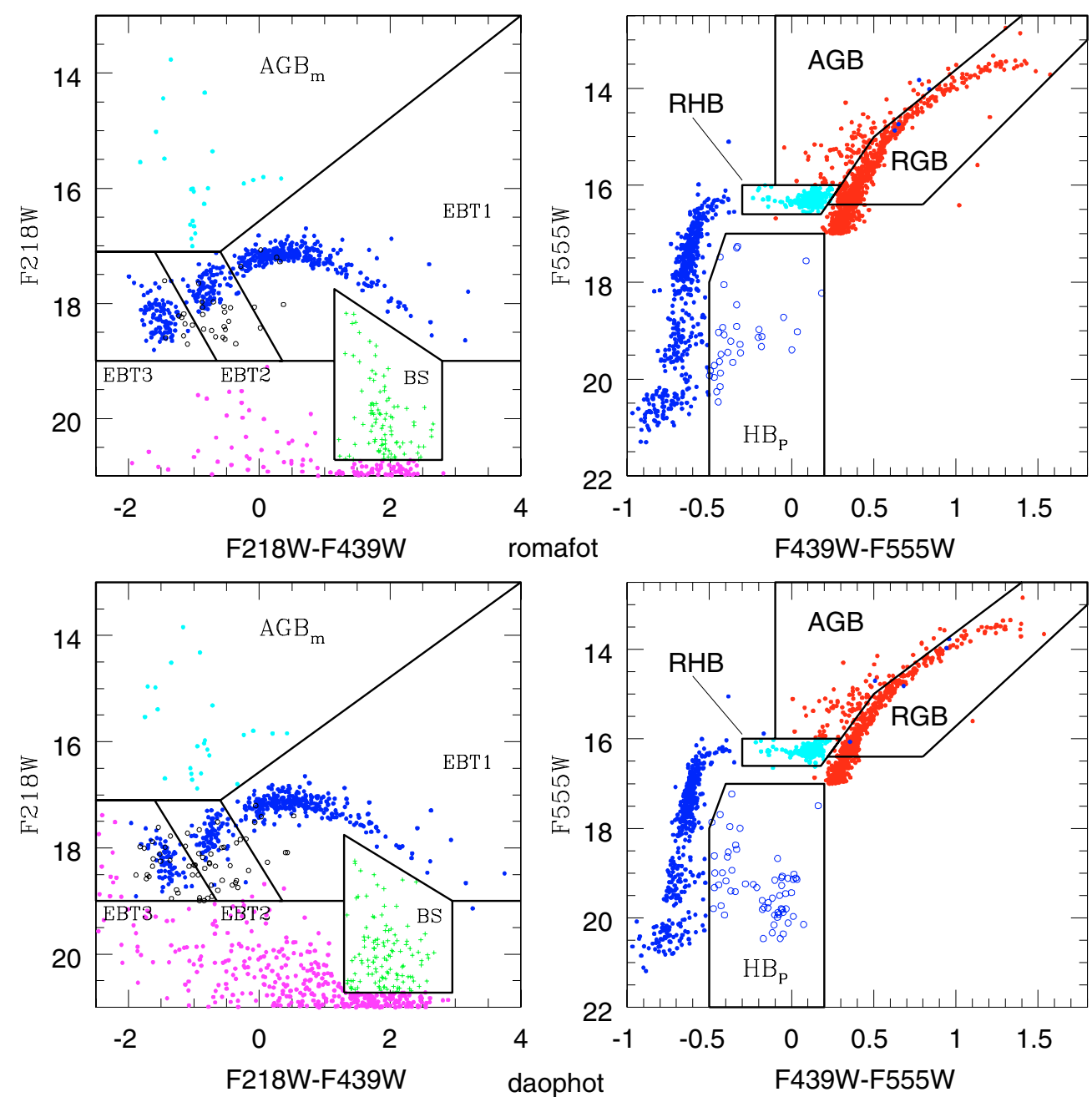

Fig. 2. Selected regions of the UV (right) and optical (left) CMDs. Different boxes show magnitude and color ranges adopted to select the different stellar samples (see labels). Upper and lower panels display photometric data reduced using ROMAFOT and DAOPHOT, respectively. Note that MS, sub-giant-branch, BS and faint RGB stars have been artificially removed to make clearer the selection among HB and RGB stars.(This figure is available in colour in electronic form.)

much larger in the CMD obtained using DAOPHOT. However, in the bright region the different CMDs appear quite similar, with clear evidence for the already known trichotomy of blue HB stars.

\section{Horizontal Branch structures}

The opportunity to analyse, for the same cluster, both UV and optical data allow us to perform a detailed analysis of luminous cluster stars in the various evolutionary phases. Figure 2 shows the boxes used to select the different samples. Blue HB stars, following Bedin et al. (2000), have been divided into three groups, EBT1, EBT2 and EBT3 in order of increasing effective temperature. In particular, the EBT1 sample includes hot HB stars located between the blue edge of the RR Lyrae instability strip and the first gap; the EBT2 sample includes extreme HB stars located between the first and the second gap; the EBT3 sample includes extreme HB stars hotter than the second gap; the AGB - manque' $\left(\mathrm{AGB}_{\mathrm{m}}\right)$ sample includes low-mass AGB stars brighter than typical HB stars. These subsamples and Blue Straggler (BS) stars (plus signs) can be easily identified in the $F 218 W, F 218 W-F 439 W$ diagram (left panels), since in this plane they are brighter and cover a color range of approximately $5 \mathrm{mag}$. For the same reasons, red HB (RHB) stars, RGB stars and AGB stars were selected in the $F 555 \mathrm{~W}$, $F 439 W-F 555 W$ diagram. The conclusions of this investigation do not depend on the criteria adopted to select individual HB and RGB subsamples.

However, Figs. 1 and 2 reveal the presence of a peculiar group of objects, which in the $F 218 W, F 218 W-F 439 W$ CMD behave either as regular or as subluminous EBT2 or EBT3 stars, whereas in the optical CMD they appear systematically cooler than typical HB stars. We will call these peculiar objects HBp stars, marking their position in the CMD with open circles (see Fig. 2). The HBp region shown in Fig. 2 (left panels) would include also all the BSs population, not shown here. We call HBp, however, only those stars that lie in the BSS region in optical CMDs, but near the HB in the UV ones (see right panels of Fig. 2), where the distinction between BSs and HBp is unambiguous. This behavior is indicative of the composite nature of the spectrum and can be caused either by photometric blends or by physical binarity (see, e.g., Allard et al. 1994). A detailed check of individual 


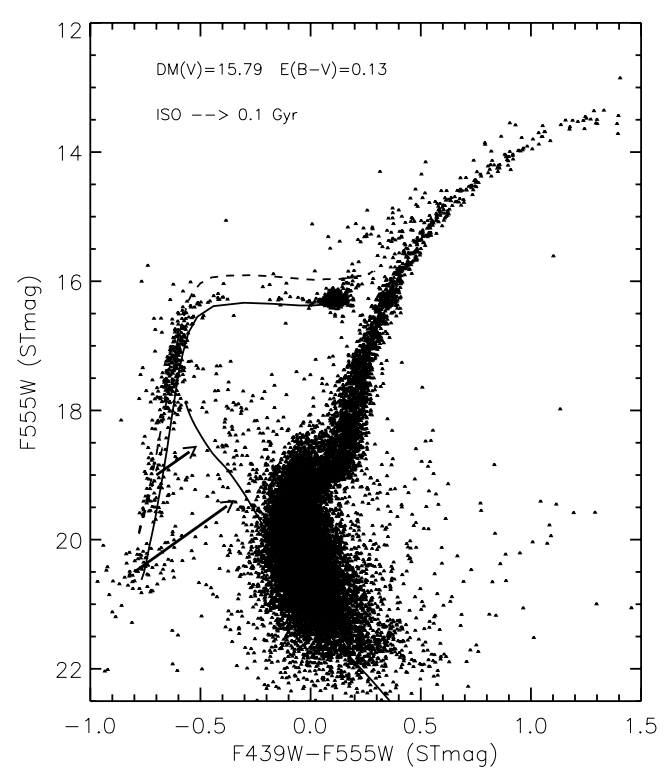

Fig. 3. The best fit of theoretical predictions to the observed $F 555 W$, $F 439 W-F 455 W$ CMD. The photometry was performed with DAOPHOT. The solid and the dashed line located below and above $\mathrm{HB}$ stars display the ZAHB and the exhaustion of central He for $Z=0.001$ and $Y=0.23$. The solid line shows a stellar isochrone for the same chemical composition and an age equal to $0.1 \mathrm{Gyr}$. The arrows show the shift in color and in magnitude of different HB stars due to either a companion or a blend with a MS star. (This figure is available in colour in electronic form.)

objects on optical and UV images, performed with ROMAFOT, shows that the group of cooler HBp $(F 555 W \approx 20.0-20.5$, $F 439 W-F 555 W \approx-0.2-0.0$ ) objects detected by DAOPHOT is mainly due to blends, i.e. the faint companions located close to these stars have not been detected. However, the surviving HBp objects appearing in the ROMAFOT CMD have very regular images, and we believe that it is rather improbable that they could be the result of chance blends with foreground stars. This hypothesis is also supported by the evidence that HBp stars are distributed across the cluster core and do not show a centrally peaked distribution. Therefore, HBp stars might be considered as a possible evidence for a binary nature of at least a fraction of EBT objects.

For reference, the arrows plotted in Fig. 3 show the shift in the CMD position for a typical EBT3 or EBT2 stars, caused by a blend with a redder companion. To mimic the most plausible events we assumed that the blended star (or the companion star in case of a physical binary) is a Main Sequence (MS) star located just below the Turn Off, namely at $F 555 W \sim 20$ and $F 439 W-F 555 W \sim 0.0$. Not surprisingly, one finds a maximum shift in the faintest EBT3 objects, a much smaller effect in the EBT2 group and virtually no shift at all among stars in the EBT1 group. The distribution of observational data convincingly indicates that the large majority of $\mathrm{HBp}$ objects indeed originated from the EBT3 group. At least a part of the spread in both EBT2 and EBT3 groups is due either to binaries or to blends. In the hypothesis of binaries, this affects photometric data only when the companion of the hot HB stars is a relatively bright $\mathrm{H}$ burning structure. Thus one could not exclude that a much larger fraction of EBT3 objects might be binaries. To constrain this working hypothesis on a more quantitative basis, we performed several artificial star experiments. In order to evaluate the completeness of hot HB stars we estimated the ridge line of this sample in the $F 218 W-F 439 W, F 218 W$ plane (see Fig. 1) and then we randomly distributed 150 artificial stars on each WFPC2 chip. The photometry on individual images was performed with ROMAFOT following the same reduction strategy adopted to obtain the original photometry. We found that the completeness is higher than $99 \%$ over the entire magnitude range $(16.8 \leq F 218 \mathrm{~W} \leq 20)$. The same procedure was adopted to estimate the completeness of HB and RGB stars brighter than $F 555 W \leq 17$ in the $F 439 W-F 555 W, F 555 W$ plane (see Fig. 1). The main difference is that the number of artificial stars was increased to 400 per WFPC 2 chip. We found that the completeness is higher than $99 \%$ for $F 555 W \leq 17$ and becomes equal to $87 \%$ in the magnitude range $20 \leq F 555 \mathrm{~W} \leq$ 21 . According to these findings we did not account for uncertainty due to completeness, since the selection of HB and RGB subsamples in different CMDs presents a completeness on average better that $99 \%$. Moreover and even more importantly, we found that the fraction of HB stars recovered inside the HBp region of the $F 439 W-F 555 W, F 555 W$ plane (see Fig. 2) is less than $2 \%$ of the total number of artificial HB stars adopted in the experiment (16 vs. 1000). This finding would imply that the fraction of blends in the sample of $\mathrm{HB}_{\mathrm{p}}$ stars is at most of the order of $50 \%$.

For comparison with theoretical predictions we will rely on the recent set of HB models by Cassisi et al. (2004), by assuming an original cluster composition of $Z=0.001$ and $Y=0.23$ (Harris 1996, but see also Bedin et al. 2000 for a discussion on the cluster metallicity). Figure 3 shows the best fit of the optical CMD as obtained for the distance and reddening indicated in the labels. We attempted a simultaneous fit of the UV CMD (Fig. 4) using the same distance and reddening, and converting the $E(B-V)$ to the corresponding extinction in $F 218 \mathrm{~W}$ through the reddening law given in Cardelli et al. (1989). However, as already found by Sosin et al. (1997), such a value did not provide a proper match between theory and observations. A reasonable fit to the UV CMD (see Fig. 4) requires a small adjustment in reddening, namely $A_{F 218 W} / E(F 439 W-F 555 W)=3.60$, slightly larger than the value given by Cardelli et al. (3.23), and a distance modulus $(m-M)_{O}=15.42$, which is only 0.03 mag larger than the one in Fig. 3.

However, in both Figs. 3 and 4 one may appreciate the good agreement between observed and theoretical HB sequences. In particular, Fig. 4 greatly improves the UV picture of HB stars in NGC 2808 already given by Sosin et al. (1997), Brown et al. (2001), and Moehler et al. (2004), since the present CMD covers, for the first time, the entire temperature range of HB stars, i.e. from red $\mathrm{HB}$ to the extreme blue tail and with sizable samples of both EBT2 and EBT3 groups. The morphology of the hot end of HB stars has already been addressed in several relevant theoretical and empirical investigations. D'Cruz et al. (1996) first advanced the suggestion of a connection between the hotter HB structures at the predicted occurrence of "hot flashers", namely the stars that ignite $\mathrm{He}$ after leaving 
Table 1. Selected parameters for the hot end of the theoretical ZAHB with $Z=0.001$ and $Y=0.23$. The mass of the He-core is $M_{\mathrm{c}}=0.5018 M_{\odot}$.

\begin{tabular}{ccccccc}
\hline \hline$M$ & $M_{\text {env }}$ & $T_{\mathrm{e}}$ & $F 218 W$ & $F 218 W-F 439 W$ & $F 218 W-F 555 W$ & $F 439 W-F 555 W$ \\
\hline 0.5022 & 0.0004 & 33788 & 1.4847 & -2.448 & -3.348 & -0.899 \\
0.5028 & 0.0010 & 31860 & 1.4513 & -2.372 & -3.260 & -0.888 \\
0.5040 & 0.0022 & 31160 & 1.4273 & -2.339 & -3.224 & -0.884 \\
0.5060 & 0.0042 & 30320 & 1.3881 & -2.301 & -3.180 & -0.879 \\
0.5080 & 0.0062 & 29066 & 1.3384 & -2.243 & -3.115 & -0.871 \\
0.5100 & 0.0082 & 27742 & 1.2949 & -2.177 & -3.043 & -0.866 \\
0.5150 & 0.0132 & 25385 & 1.1947 & -2.046 & -2.896 & -0.850 \\
0.5200 & 0.0182 & 23762 & 1.1231 & -1.944 & -2.783 & -0.839 \\
0.5250 & 0.0232 & 22469 & 1.0553 & -1.853 & -2.684 & -0.830 \\
0.5300 & 0.0282 & 21385 & 0.9951 & -1.770 & -2.593 & -0.823 \\
0.5350 & 0.0332 & 20480 & 0.9487 & -1.695 & -2.510 & -0.816 \\
0.5400 & 0.0382 & 19542 & 0.8962 & -1.620 & -2.429 & -0.809 \\
0.5450 & 0.0432 & 18933 & 0.8572 & -1.551 & -2.354 & -0.803 \\
0.5500 & 0.0482 & 18249 & 0.8127 & -1.478 & -2.276 & -0.797 \\
0.5600 & 0.0532 & 17021 & 0.7285 & -1.335 & -2.120 & -0.786 \\
0.5700 & 0.0632 & 15929 & 0.6506 & -1.188 & -1.963 & -0.775 \\
0.5800 & 0.0732 & 14753 & 0.5545 & -1.005 & -1.672 & -0.763 \\
\hline
\end{tabular}

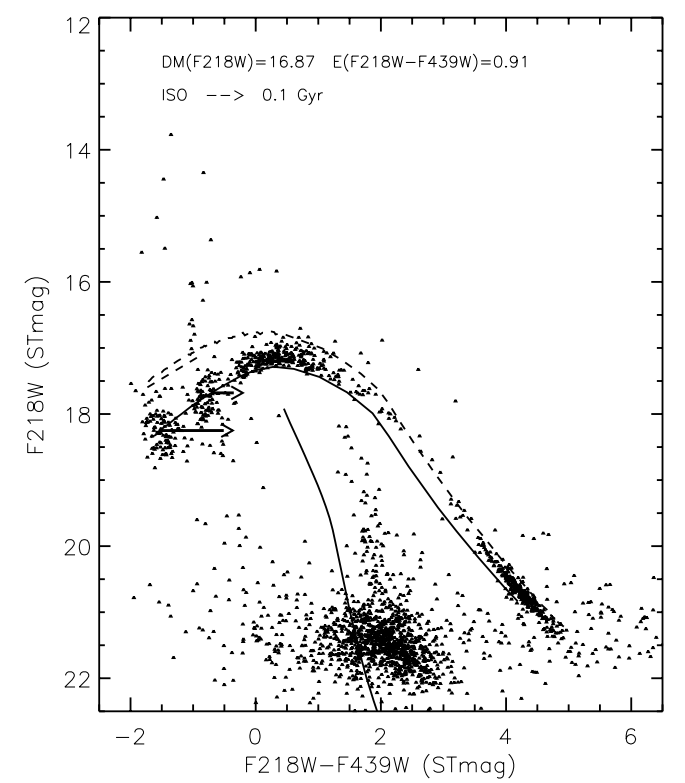

Fig. 4. Same as in Fig. 3 but for the $F 218 W, F 218 W-F 439 W$ CMD. The photometry was performed with ROMAPHOT.

the Red Giant Branch due to a huge mass loss. By following Brown et al. (2001, but see also Cassisi et al. 2003a) the EBT3 group should be the result of Helium flash-induced mixing, enriching the envelopes of $\mathrm{He}$ and $\mathrm{C}$ and pushing the star at effective temperatures beyond the canonical end of the Horizontal Branch and at fainter magnitudes. Spectroscopic investigations by Moehler et al. (2004) have recently supported such a picture, showing that EBT3 stars are both hotter and more He-rich than canonical HB stars, although revealing an unexpected presence of atmospheric H. According to Unglaub (2004) the occurrence of atmospheric $\mathrm{H}$ might be due to a mixing of processed material to the stellar surface and to the upward diffusion of $\mathrm{H}$ during subsequent evolutionary phases.

In this context the current data raise several questions. According to the quoted scenario, the canonical ZAHB should stop at the temperature of EBT2 stars, but Sosin et al. (1997) already presented a ZAHB approaching the effective temperatures typical of EBT3 stars. Brown et al. (2001) pointed out the problem and claimed that this is the result of the adopted ZAHB which reaches envelope masses as small as $10^{-4} M_{\odot}$, i.e. well below the true termination of the canonical ZAHB. According to Brown et al. (2001) the canonical ZAHB should have a lower limit in the envelope mass equal to $M_{\text {env }} \sim 0.0006 M_{\odot}$. Such a limit could be much less firm than currently assumed, since Brown et al. (2001) and D'Cruz et al. (1996) have explicitly neglected mass loss in the post RGB evolution.

However, to further constrain this point we artificially truncated the canonical ZAHB at an envelope mass that is almost a factor of two larger than the quoted lower limit $\left(M_{\text {env }} / M_{\odot} \sim\right.$ 0.001 vs. 0.0006$)$. Data plotted in Fig. 4 clearly show that the canonical ZAHB is, against the expectation, still reaching the EBT3 group. To look into this problem in some detail, Table 1 gives our theoretical predictions for the hot end of the ZAHB distribution. Comparison of these values with the observed distribution in Fig. 4 reveals that the blue boundary of the EBT1 should lie at $T_{\mathrm{e}} \sim 16000 \mathrm{~K}$, whereas EBT2 structures appear in the interval $18000 \leq T_{\mathrm{e}} \leq 23000 \mathrm{~K}$. Such a prediction appears in agreement with the spectroscopic temperatures determined by Moehler et al. (2004) for three EBT2 structures, namely 20100,21100 and $21300 \mathrm{~K}$. According to current HB models, stellar strucutures at the blue end of the EBT2 region should still have an envelope as large as $M_{\mathrm{env}} \approx 0.01 M_{\odot}$, i.e., much larger than the quoted lower limit of $0.0006 M_{\odot}$. At this limit our models reach an effective temperature of $T_{\mathrm{e}} \sim 32000 \mathrm{~K}$, well beyond the red boundary of the EBT3 group, which lies at $T_{\mathrm{e}} \sim 28000 \mathrm{~K}$.

Moreover, Fig. 4 shows that a large fraction of both EBT2 and EBT3 groups falls below the theoretical ZAHB. The underluminosity of EBT3 structures has been often considered as empirical evidence supporting the mixing scenario, which -however- should not affect the EBT2 group. In this paper we have shown that such an underluminosity might be partially due to binarity (or blending). To reach firm conclusions one 
Table 2. Star counts of Red Giant Branch, Horizontal Branch, Asymptotic Giant Branch and Blue Straggler stars according to the photometry performed with ROMAFOT.

\begin{tabular}{|c|c|c|c|c|c|c|c|c|c|c|c|c|}
\hline$r$ & RGB & RHB & EBT1 & EBT2 & EBT3 & $\mathrm{HBp}$ & $\mathrm{HB}$ (tot) & $R$ & $\overline{\sigma_{R}}$ & AGB & $\mathrm{AGB}_{\mathrm{m}}$ & $\overline{\mathrm{BS}}$ \\
\hline \multicolumn{13}{|l|}{ HST - ROMAFOT } \\
\hline $0.0-15.6$ & 163 & 113 & 64 & 22 & 17 & 8 & 224 & 1.37 & 0.14 & 33 & 2 & 43 \\
\hline $15.6-45.6$ & 226 & 160 & 119 & 21 & 26 & 16 & 342 & 1.51 & 0.13 & 30 & 8 & 32 \\
\hline $45.6-120$ & 199 & 123 & 124 & 50 & 45 & 10 & 352 & 1.77 & 0.16 & 19 & 12 & 22 \\
\hline TOTAL & 588 & 396 & 307 & 93 & 88 & 34 & 918 & 1.56 & 0.08 & 82 & 22 & 97 \\
\hline \multicolumn{13}{|l|}{ Bedin et al. (1999) } \\
\hline $120-160$ & 92 & 68 & 34 & 23 & 32 & $\ldots$ & 157 & 1.71 & 0.22 & 16 & $\ldots$ & . . \\
\hline $160-250$ & 84 & 82 & 32 & 18 & 32 & $\ldots$ & 164 & 1.95 & 0.26 & 21 & $\ldots$ & .. \\
\hline TOTAL Bedin & 176 & 82 & 150 & 41 & 64 & $\ldots$ & 321 & 1.82 & 0.17 & 37 & $\ldots$ & $\cdots$ \\
\hline ROMAFOT+Bedin & 764 & 478 & 457 & 134 & 152 & $\ldots$ & 1239 & 1.62 & 0.07 & 119 & $\ldots$ & $\ldots$ \\
\hline
\end{tabular}

should have a better insight into the role of binarity in both the location in the CMD and in the spectral features. The distribution in Fig. 4 appears poorly in agreement with the predictions of the mixing scenario, as suggested by data plotted in Fig. 4 (panel b) of Brown et al. (2001). We conclude that the problem of hot HB stars in NGC 2808 is far from being settled, and deserves further accurate investigations. Current findings support the alternative hypothesis, originally suggested by Bailyn \& Iben (1989), that cluster sdB stars might be the aftermath of mergers of low-mass helium white dwarfs formed from primordial main sequence binaries.

The Blue Straggler sequence $(F 218 W \leq 21,1.5 \leq$ $F 218 W-F 439 W \leq 2.5$ ) follows the predicted $0.1 \mathrm{Gyr}$ isochrone, but clearly shifted toward redder colors. This occurrence can also be detected in the STIS UV CMD provided by Brown et al. (2001, see their Fig. 3). For a detailed discussion concerning the evolutionary properties of cluster BSs see Piotto et al. (2004).

\section{Star counts and population gradients}

To properly investigate the radial distribution of the different samples we split the field covered by WFPC2 in concentric annuli. The annuli were selected at the core radius (15.6 arcsec), at the half-mass radius (45.6 arcsec) and to the edge of the WF chip, namely 120 arcsec. Structural parameters for NGC 2808 come from the compilation by Harris (1996). Table 2 gives star counts for the various selected phases and in the three selected annuli. In the same table current data were complemented with star counts based on ground-based $U$ and optical $B, V$ data collected by Bedin et al. (2000). Note that to avoid spurious contaminations from field stars we restricted the star counts to two annuli that range from 2 to roughly 4 arcmin from the cluster center (see Fig. 16 in Bedin et al. 2000). As a result, we are dealing with a sample of more than $1200 \mathrm{HB}$ stars, i.e. one of the most statistically significant sample of HB stars in GGCs.

To determine whether the evolved population presents any peculiar trend we estimated the $R$ and the $R^{\prime}$ parameters, i.e. $R=N_{\mathrm{HB}} / N_{\mathrm{RGB}}, R^{\prime}=N_{\mathrm{HB}} /\left(N_{\mathrm{RGB}}+N_{\mathrm{AGB}}\right)$ where the sample of cool giants refers to structure more luminous that the HB luminosity level. The HB luminosity was fixed $2 \sigma$ below the peak in the luminosity distribution of red HB stars,

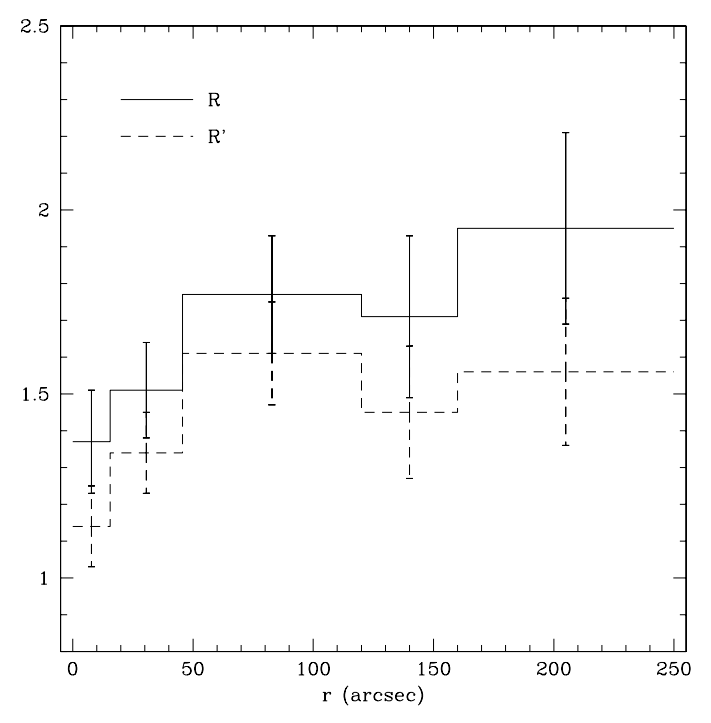

Fig. 5. The parameters $R$ (solid line) and $R^{\prime}$ (dashed line) as a function of the radial distance according to ROMAFOT photometry.

i.e. $F 555 W=16.4$ mag. Figure 5 shows the radial distribution of $R$. Data plotted in this figure show that these parameters steadly increase when moving from the center to the outermost regions, passing from $1.37 \pm 0.14$ in the cluster core to $1.51 \pm 0.13$ at half-mass radius and to $1.95 \pm 0.26$ in the outer regions. This finding is independently supported by star counts based on the DAOPHOT/ALLFRAME cluster photometry (see data listed in Table 3).

Note that in the outermost regions, ground-based photometry is missing the HBp group, thus underestimating the actual number of HB structures. This means that the two outermost $R$ values should be considered as (marginally) underestimated. Figure 5 shows the presence of a population gradient, for which the number ratio between HB and RGB stars increases when moving from the center toward the cluster periphery. This finding supports the color gradient detected by Sohn et al. (1998). In particular, they found that the cumulative color distribution ranges from $U-B=0.4$ at the center of the cluster to $U-B=0.2$ at $r \geq 70 \operatorname{arcsec}$ (see their Fig. 1). We mentioned that both Walker (1999) and Bedin et al. (2000) did not detect a well-defined population gradient. However, Walker by using accurate ground-based data found that the ratio of AGB 
Table 3. Star counts of Red Giant Branch, Horizontal Branch, Asymptotic Giant Branch and Blue Straggler stars according to the photometry performed with DAOPHOT/ALLFRAME.

\begin{tabular}{|c|c|c|c|c|c|c|c|c|c|c|c|c|}
\hline$r$ & RGB & $\overline{\mathrm{RHB}}$ & EBT1 & EBT2 & EBT3 & HBp & HB(tot) & $\bar{R}$ & $\overline{\sigma_{R}}$ & $\overline{\mathrm{AGB}}$ & $\mathrm{AGB}_{\mathrm{m}}$ & BS \\
\hline \multicolumn{13}{|l|}{ HST - DAOPHOT } \\
\hline $0.0-15.6$ & 154 & 110 & 67 & 17 & 16 & 14 & 224 & 1.45 & 0.15 & 35 & 2 & 51 \\
\hline $15.6-45.6$ & 213 & 158 & 127 & 26 & 21 & 20 & 352 & 1.65 & 0.14 & 31 & 9 & 44 \\
\hline $45.6-120$ & 181 & 124 & 95 & 32 & 35 & 26 & 312 & 1.73 & 0.16 & 20 & 13 & 24 \\
\hline TOTAL & 548 & 392 & 289 & 75 & 72 & 60 & 888 & 1.62 & 0.09 & 86 & 24 & 119 \\
\hline DAOPHOT+Bedin & 724 & 474 & 439 & 116 & 136 & $\ldots$ & 1209 & 1.67 & 0.08 & 123 & $\ldots$ & $\ldots$ \\
\hline
\end{tabular}

plus RGB stars brighter than $V=15$ with the RGB stars with $15 \leq V \leq 17.5$ ranges from 8.8 for $r \geq 65$ arcsec, to 5.4 for $22<r<65$ arcsec, and to 1.4 for $r \leq 22$ arcmin. No firm conclusion was reached due to the crowding in the innermost cluster regions. Moreover, Bedin et al. by using both ground based and HST data did not detect a statistically significant population gradient, but a possible decrease of RGB stars for $r \leq 100$ arcsec, and a lack of EBT3 stars for $r \approx 250 \operatorname{arcsec}$ (see their Fig. 17). The main difference between the present analysis and the one by Bedin et al. is that we performed the initial search for hot HB stars in the $F 218 W$-band images, hence our completeness is higher in this filter.

The evidence for very hot HB structures, approaching the limiting mass for He ignition, can be taken as evidence that some cluster stars are expected to follow different types of peculiar evolutionary histories. A first and well-known occurrence is that very hot $\mathrm{HB}$ structures will miss their proper AGB phase, crossing the CMD as luminous "AGB-manquè" structures to reach at even higher temperatures their cooling radius as Carbon-Oxygen (CO) core white dwarfs (WDs). However, if mass loss has brought some Red Giants to the limiting mass for He ignition, it appears unlikely that the same mechanism also produced Red Giant stars below that limit. As a consequence, we foresee the additional occurrence of $\mathrm{RG}$ missing their HB progenies, directly cooling down as Hecore WDs. We note that the cooling times of He-core WDs are longer than that of CO-core WDs ${ }^{1}$. This intrinsic difference might be the crucial parameter to identify the contribution of similar structures in NGC 2808 or in other clusters with extended Blue Tails.

Bearing in mind such a scenario, one can use star counts to test the level of agreement between theory and observations. From the theoretical side, Fig. 6 summarizes the evolutionary fates of the HB structures. From evolutionary times one derives that for $\mathrm{HB}$ stars producing $\mathrm{AGB}$ progenies one expects a number ratio $N(\mathrm{AGB}) / N(\mathrm{HB}) \sim 0.11$ (see, e.g., Cassisi et al. 2004). The number ratio between AGB-manquè and their hot HB progenitors should again be of the order of 0.10 . As far as the empirical measurements is concerned, one finds that both RHB and EBT1 groups should enter the AGB phase, whereas EBT2, EBT3 and HBp should produce AGB-manquè structures before cooling down as WDs. A glance at the data listed

${ }_{1}$ The lifetime for a typical CO-core WD with $M=0.5 M_{\odot}$, and $M_{B} \sim 11.5$ is equal to $t \approx 3.5 \times 10^{8} \mathrm{Myr}$, while for a He-core WD with $M=0.3 M_{\odot}$, and $M_{B} \sim 11.5$ is roughly a factor of two longer $\left(t \approx 6.7 \times 10^{8} \mathrm{Myr}\right)$.

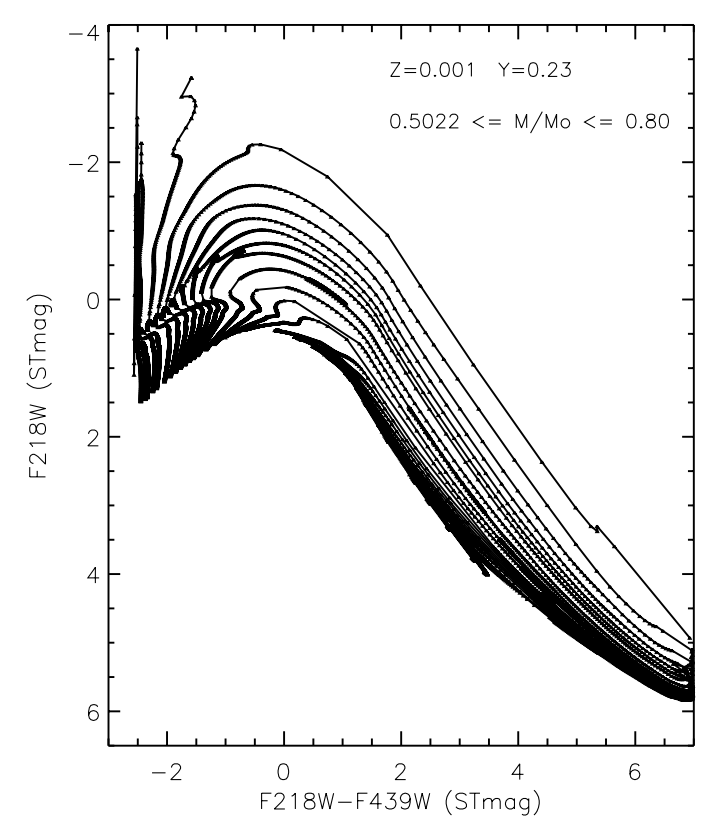

Fig. 6. The predicted evolutionary tracks from the ZAHB of the adopted stellar models.

in Table 1 shows that $N(\mathrm{AGB}) / N(\mathrm{HB})=0.116 \pm 0.01$ and $N\left(\mathrm{AGB}_{\mathrm{m}}\right) / N(\mathrm{HB})=0.102 \pm 0.02$, and therefore in good agreement with current predictions.

Ome may finally notice that the number ratio between BS and HB stars is of the order of 0.1 , thus confirming the relative lack of BSs found by Piotto et al. (2004) in clusters with total luminosity larger than $M_{V} \leq-9$.

\section{HB gradients, Blue Tails and the second parameter: a discussion}

The evidence for the possible occurrence of binary systems, at least in the EBT3 group, appears to us as the most relevant outcome of this investigation. It suggests that binarity could be the origin of the huge mass loss which removed from their RG progenitors a substantial fraction of their H-rich envelope. It is true that in the present photometry only a few extreme HB stars show the fingerprint of binarity, but MS companions significantly fainter than TO stars would not produce significant shifts in the magnitude of HB stars. Therefore, extremely hot cluster HB stars behave like their Galactic field counterparts, the sdB stars, where the binarity plays a relevant role (see Han et al. 2003, and references therein). In this context one could speculate a possible scenario accounting for the peculiar 
trichotomy of the blue HB. Stars in the EBT1 group come from a mass loss mechanism acting on single stars. Hotter EBT2 and EBT3 stars, or at least a fraction of them, have suffered greater mass loss, possibly due to mass exchange with a companion that pushes these stars toward the hot end of the HB. However, in the case of extreme mass loss, stars will ignite $\mathrm{He}$ as "hot flashers" spending their HB lifetime as a separate group of stars (EBT3) at the hot end of the normal HB (Sweigart 1997; Momany et al. 2004).

As far as the occurrence of radial gradients is concerned, an expanded spatial distribution of EHB stars has already been found in a few GGCs, such as $\omega$ Cen (D'Cruz et al. 2000) and NGC 6101 (Marconi et al. 2001). If one assumes that the gradient of $R$ in NGC 2808 is real, one can further speculate about the possible origin of blue HB stars. Since Iben (1968), the $R$ parameter has been widely used as an indicator of the original He content of HB progenitors. Even though the theoretical calibration of $R$ in terms of the original helium is far from being firmly settled (see, e.g. Cassisi et al. 2003b), the most straightforward interpretation of the $R$ gradient would be in terms of He: the original He content of HB stars increasing from the center to the cluster periphery. However, an original spatial gradient in the He abundance is unlikely, as is its survival over the many Gyrs of the cluster lifetime. Therefore we believe that the He gradient hypothesis has to be ruled out. Similarly, the dynamical relaxation of the less massive HB stars cannot be invoked, because it has been repeatedly shown that the HB lifetimes are not long enough.

Bearing in mind that $\mathrm{HB}$ stars are the progenies of RG stars, the two populations must have the same distribution, unless something happened at the passage from RG to HB structures. Thus one should conclude that something has produced a huge amount of mass loss in some RG stars, at the same time ejecting these progenitors outside the cluster core. This could be a continuous process, refurbishing continuously the cluster halo with BHB. However, one could connect such a mechanism with a past episode of core collapse, when the tidal stripping of RG may be suddenly and enormously enhanced. The new born hot HB stars would be rapidly thermalized, the actual spatial distribution possibly being a signature of this dynamical event. In this sense, we are not sure that the expanded distribution necessarily argues against a dynamical origin, as claimed by D'Cruz et al. (2000).

As recently pointed out by Rosenberg et al. (2004) discussing the case of M54, the most striking and undeniable evidence concerning Blue Tails is that they appear in globulars which are among the most massive clusters in our Galaxy (see also Moehler et al. 2004). However, not all massive clusters have Blue Tails. If cluster dynamics plays a significant role, one should conclude that Blue Tails are a transient phenomenon rather than a continuous one. The occurrence of this phenomenon might be connected with the already-mentioned core collapse, a dynamical phase during which stellar interactions and tidal stripping have great efficiency. Against such an hypothesis, one could invoke the evidence that NGC 2808 does not show the typical signature of post-core-collapsed clusters (Djorgovski et al. 1991; Fusi Pecci et al. 1993). However, Meylan \& Heggie (1997) have already stressed that the separation between "King model clusters" and "core-collapsed clusters" may often be less clear-cut than generally believed, since we do not exactly know how a cluster appears between two episodes of collapse. Moreover, one may put the problem on very general grounds recalling, again from Meylan \& Heggie (1997), "the outstanding problem implied by the many clusters - like 47 Tuc - which show no trace of core-collapse, but have short enough dynamical times for collapsing in a short fraction of the Hubble time".

According to such a picture, we suggest that Blue Tails and the second parameter problem could be quite different evolutionary features. The former is likely connected to the dynamical interaction in massive clusters, whereas the latter is possibly connected to an age difference as recently dicussed by Rey et al. (2001) for M 3 and M 13.

\section{Final remarks}

We have already quoted the uncertainties affecting the theoretical calibration of the $R$ parameter in terms of the original helium content and the accompanying uncertainty on the empirical estimates. Recent investigations indicate for NGC 2808 an $R$ parameter equal to $0.99 \pm 0.1$ (Sandquist 2000) or $R=1.26 \pm$ 0.06 (Zoccali et al. 2000) against the value $R=1.62( \pm 0.07)$ derived in this paper. It appears that current estimates of statistical errors for the $R$ parameter are of minor relevance when compared with completeness errors of the sample, at least when dealing with clusters with extended Blue Tails.

Bearing in mind the caveat concerning theoretical calibrations, we note the good agreement of present $R$-value with the most recent calibration given by Cassisi et al. (2003), which for $[\mathrm{M} / \mathrm{H}] \sim-1.03$ predits $R \sim 1.55$ (Zoccali et al. 2000). However, that calibration is adequate for clusters with HB structures not hotter than the RR Lyrae instability strip, since evolutionary times of very blue HB structures can be up to about $40 \%$ longer (Cassisi et al. 2004). However, according to the evidence that EBT2 and EBT3 stars are approximately the $30 \%$ of the entire HB sample, the value of the above quoted calibration should be increased by no more than $19 \%$, thus improving the agreement between theory and observations. This, in our opinion, can be taken as independent support of the ${ }^{12} \mathrm{C}+\alpha$ reaction rate adopted in the theoretical calibration.

Acknowledgements. It is a pleasure to thank C.E. Corsi, I. Ferraro and L. Pulone for several useful suggestions concerning the artificial star experiments. We also acknowledge Dr. Ivo Saviane as referee for his pertinent comments that helped us to improve the content and the readability of the manuscript. This work was partially supported by MIURCOFIN 2002 under the project "Stellar Populations in Local Group Galaxies" and MIUR-COFIN 2003 under the project "Continuity and Discontinuity in the Galaxy Formation". M.Z. thanks Giampaolo Piotto for providing financial support and computer facilities.

\section{References}

Allard, F., Wesemael, F., Fontaine, G., Bergeron, P., \& Lamontagne, R. 1994, AJ, 107, 1565

Bailyn, C. D., \& Iben, I. Jr. 1989, ApJ, 347, L21

Bedin, L. R., Piotto, G., Zoccali, M., et al. 2000, A\&A, 363, 159 
Brown, T. M., Sweigart, A. V., Lanz, T., Landsman, W. B., \& Hubeny I. 2001, ApJ, 562, 368

Buonanno, R., \& Iannicola, G. 1989, PASP, 101, 294

Cardelli, J., Clayton, G. C., \& Mathis, J. S. 1989, ApJ, 345, 245

Cassisi, S., Schlattl, H., Salaris, M., \& Weiss, A. 2003a, Apj, 582, L46

Cassisi, S., Salaris, M., \& Irwin, A. W. 2003b, ApJ, 588, 862

Cassisi, S., Castellani, M., Caputo, F., \& Castellani, V. 2004, in press

D'Cruz, N., Dorman, B., Rood, R. T., \& O'Connel, R. W. 1996, ApJ, 466, 359

D’Cruz, N. L., O'Connel, R. W., Rood, R. T., et al. 2000, ApJ, 530, 352

Djorgovski, S., Piotto, G., Phinney, E. S., \& Chernoff, D. F. 1991, ApJ, 372, L41

Ferraro, F. R., Paltrinieri, B., Rood, R. T., \& Dorman B. 1999, ApJ, 522, 983

Fusi Pecci, F., Ferraro, F. R., Bellazzini, M., et al. 1993, AJ, 105, 1145

Han, Z., Podsiadlowski, Ph., Maxted, P. F. L., \& Marsh, T. R. 2003, MNRAS, 341, 669

Harris, W. E. 1974, ApJ, 192, L161

Harris, W. E. 1978, PASP, 90, 45

Harris, W. E. 1996, AJ, 112, 1487

Holtzman, J. A., Burrows, C. J., Casertano, S., et al. 1995, PASP, 107, 1065
Iben, I. Jr. 1968, Nature, 220, 143

Marconi, G., Andreuzzi, G., Pulone, L., et al. 2001, A\&A, 380, 478

Meylan, G., \& Heggie, D. C. 1997, A\&AR, 8, 1

Moehler, S., Sweigart, A. V., Landsman, W. B., Hammer, N. J., \& Dreizler S. 2004, A\&A, 415, 313

Momany, Y., Bedin, L. R., Cassisi, S., et al. 2004, A\&A, 420, 605

Piotto, G., King, I. R., Djorgovski, S. G., et al. 2002, A\&A, 391, 945

Piotto, G., De Angeli, F., King, I. R., et al. 2004, ApJ, 604, L109

Rey, S.-C., Yoon, S.-J., Lee, Y.-W., Chaboyer, B., \& Sarajedini, A. 2001, ApJ, 122, 3219

Rosenberg, A., Recio-Blanco, A., \& Garcia-Marin, M. 2004, ApJ, 603,135

Sandquist, E. L. 2000, MNRAS, 313, 571

Sohn, Y. J., Byun, Y. I., Yim, H. S., Rhee, M.-H., \& Chun, M.-S. 1998, JASS, 15,1

Sosin, C., Dorman, B., Djorgovski, S. G., et al. 1997, ApJ, 480, L35

Stetson, P. B. 1987, PASP, 99, 191

Stetson, P. B. 1994, PASP, 106, 250

Sweigart, A. V. 1997, ApJ, 474, L23

Unglaub, K. 2004, in 14th European Workshop on White Dwarfs, ed. S. Moehler, \& D. Koester (San Francisco: ASP), in press

Walker, A. R. 1999, ApJ, 118, 432

Zoccali, M., Cassisi, S., Bono, G., et al. 2000, ApJ, 538, 289 